\title{
Strong localization of the density of power losses in Type-II Superconducting Wires
}

\author{
H. S. Ruiz, A. Badía-Majós ${ }^{1}$ and Y. A. Genenko, S. V. Yampolskii ${ }^{2}$ \\ ${ }^{1}$ Materials Science Institute of Aragón (ICMA-CSIC), \\ Department of Condensed Matter Physics, University of Zaragoza, Spain \\ ${ }^{2}$ Fachgebiet Materialmodellierung, Technische Universität Darmstadt, Germany
}

\begin{abstract}
A round straight superconducting wire has been used as numerical prototype for pursuing a comprehensive study on the local distribution of current and power density losses, attained by the concomitant action of an ac transport current and an oscillating transverse magnetic field. The numerical simulations have been performed within the magnetoquasisteady approach of the critical state theory, including virgin and premagnetized wires. A wide variety of shapes for the flux fronts characterizing the local dynamics of the electromagnetic quantities across the section of the wire has been revealed. Under special conditions, flux fronts characterized either by the so-called "field-like", or "current-like" shapes are shown, with the occurrence of multiple domains of current flow detached by thin lines acting as boundaries between the critical values $I_{c}$ and $-I_{c}$. Despite the lack of symmetry for attaining at least an intuitive definition on the shape of the flux front, a universal pattern of the distribution of magnetic flux has been identified. Also, a strong asymmetric localization of the power density losses has been envisaged, as long as synchronous electromagnetic excitations are used.
\end{abstract}




\section{INTRODUCTION}

Major features of the macroscopic electromagnetic behavior of type-II superconducting wires have been captured by Bean's model of the critical state ${ }^{1,2}$. In this framework, magnetization currents of density $\mathbf{J}$ are induced within the superconductor during variations of the magnetic flux which accordingly redistribute themselves to screen the penetrating flux within the sample. Their magnitude adopts the critical value $J_{c}$ at a given temperature and specified field, where all the electromagnetic quantities are supposed to be averaged over a volume containing a big enough number of vortices. Although simple for idealized configurations, the electrodynamics underlying Bean's model becomes cumbersome when realistic configurations are addressed. In fact, for most of the practical cases, the macroscopic observables such as the magnetic moment curve or the ac-losses can only be calculated through the use of sophisticated numerical methods capable of determining the time-dependent dynamics of the local current density profiles, and/or the profiles of magnetic flux density, as well as of the distribution of electric field inside of the superconducting wire.

In idealized configurations such as infinite slabs under the action of a parallel magnetic field or a longitudinal transport current, Ampere's law takes the form $d B / d x=\mu_{0} J$ and may be straightforwardly solved with some prescription for the current density $\left(|J| \leq\left|J_{c}\right|\right)$ under continuity boundary conditions that incorporate the influence of the sources. This leads to the exact analytical solution of the main experimental observables ${ }^{3}$. Being more specific, the analytical solution for these cases consists of solving a free boundary problem for the distribution of penetrating current (or magnetic flux) under a given external electromagnetic excitation. The inner flux-free region can be straightforwardly depicted by a planar front of flux for the case of a slab, or a radial flux front centered in the symmetry axis of a cylindrical wire. Thus, once a method is found for obtaining the actual size of the flux free region, all the electromagnetic observables such as the intensity of magnetic flux, the global magnetization curve, and the expected value for the ac-losses, can be deduced from the knowledge of the penetrating current profiles.

On the other hand, when realistic configurations are brought to the fore, such as a long strip with finite thickness or a bulk superconducting cylinder exposed to a transverse magnetic field, the use of computational algorithms based upon well established physical principles is unavoidable. In fact, for real applications of superconducting wires, the scenario 
is such that a simultaneous field and transport current condition must be satisfied. Then, in addition to the ambient field one has to consider the local action of the magnetic field generated by the transport current itself. Thus, as this is the configuration very often met in practice, this paper is devoted to study different configurations of oscillating transverse magnetic field and a synchronous transport current, both applied to a round superconducting wire with infinite length, prepared in a virgin or premagnetized state, with special attention to the symmetry of the flux front and the local distribution of power density losses along the cyclic stage.

\section{NUMERICAL METHOD AND THEORETICAL STATEMENTS}

In this work, the variational optimization method developed in Ref. ${ }^{4}$ has been used to iteratively solve the discretized form of Faraday's law $\delta \mathbf{B}_{i}=-\nabla \times \mathbf{E}_{i}\left(\mathbf{J}_{i}\right) \delta t$, for the problem of the long superconducting wire. The magnetoquasisteady approach $\left.\nabla \times \mathbf{B}_{i}\right)=\mu_{0} \mathbf{J}_{i}$ ) is used. In terms of the circulating current densities, the electromagnetic problem consists of solving the increment $\left.\delta \mathbf{J}_{i}\right)$ at each point of the cross section $\left(\delta \mathbf{J}=\left\{0\right.\right.$ or $\left.\left.\pm \mathbf{J}_{c}\right\}\right)$, as induced by the penetrating flus fronts.

Finite element simulations have been conducted on the basis of analytical expressions for the inductance matrices between parallel cylindrical filaments $\{i\}$, each carrying a uniform distribution of current density $J_{i}=I_{i} s_{i}$, and such that their cross sectional area $s_{i}=\pi a^{2}$ is much lesser than the cross sectional area of the entire superconducting wire $(\Omega)$. Thereby, for very large (formally infinite) filaments centered at the positions $\mathbf{r}_{i}$, the inductance matrices can be defined as

$$
\mathrm{M}_{i j}= \begin{cases}\frac{\mu_{0}}{8 \pi} & , \forall \quad r_{i}=r_{j} \in \Omega \\ -\frac{\mu_{0}}{2 \pi} \ln \left(\frac{\left|\mathbf{r}_{i}-\mathbf{r}_{j}\right|}{a}\right) & , \forall \quad r_{i} \neq r_{j} \in \Omega,\end{cases}
$$

In these expressions, arbitrary constants may be added to both matrices, which are absorbed into the customary minimization procedure of the electromagnetic Lagrange density defined for problems within the critical state theory ${ }^{4-7}$.

In practical terms, Faraday's law is replaced by a global minimization statement equivalent to evaluate the maximal projection rule $\hat{\mathbf{E}} \cdot \mathbf{J}$ over each one of the filaments filling the 
cross section of the superconducting wire ${ }^{8}$. The quantity to be minimized takes the form:

$$
\frac{1}{2} \sum_{i, j}\left\{I_{i, l+1}\left[\mathrm{M}_{i j} I_{j, l+1}+\Delta A_{0}\left(r_{i}\right)\right]-I_{i, l} \mathrm{M}_{i j} I_{j, l+1}\right\},
$$

where the set of currents $\left\{I_{i, l+1}\right\}$ defines the set of unknown variables for the time steps $l+1$, whilst an initial value defined by the set of currents $\left\{I_{i, l}\right\}$ is assumed. For a sufficiently refined mesh, the current density within each one of the filaments has to be constrained by the critical state material law $\left|I_{i}\right| \leq I_{c}$. Furthermore, the system must satisfy the additional constraint for the transport current:

$$
\sum_{s_{i} \in \Omega} I_{i}=I_{\mathrm{tr}}
$$

with $I_{\mathrm{tr}}$ the intensity of transport current injected to the superconducting wire at the time step $l+1$. Thus, specification of the time-steps for the minimization procedure may be pursued through a fine discretizing of the function $I_{t r}(t)$ when the transport current condition is recalled, or through the discretization of the vector potential component related to nonlocal sources $\mathbf{A}_{0}\left(\mathbf{r}_{i}\right)$, which in case of a uniform magnetic field $\mathbf{B}_{0}$ corresponds to $\mathbf{A}_{0}\left(\mathbf{r}_{i}\right)=$ $\mathbf{B}_{0} \times \mathbf{r}_{i}$. Notice that, in all cases the minimization principle is based upon a discretization of the path, followed by the external sources, meaning that it is an approximation to the continuous evolution whose accuracy increases as the step diminishes.

Eventually, subsequent to the minimization procedure the components of the magnetic flux density $\mathbf{B}=\nabla \times \mathbf{A}$ can be evaluated according to the definition of the total vector potential $\mathbf{A}\left(\mathbf{r}_{i}\right)=\mathbf{A}_{0}\left(\mathbf{r}_{i}\right)+\mathbf{A}_{\text {self }}\left(\mathbf{r}_{i}\right)$, where the contribution of currents derived from the local variations of the electromagnetic excitations $\left(B_{0}, I_{t r}\right)$ is given by $\mathbf{A}_{\text {self }}\left(\mathbf{r}_{i}\right)=\sum_{j} M_{i j} \mathbf{J}_{j}$.

Finally, as mentioned above, additional integration constants have been arbitrarily chosen, and the vector potential obtained is affected by this. The ambiguity may be surpassed by including the physical condition for the electric field $\mathbf{E} \equiv 0$ at those points where magnetic flux does not vary. Thus, although our statement of Faraday's law does not include the electrostatic-like term in the general expression of the electric field $\mathbf{E}=-\partial_{t} \mathbf{A}-\nabla \phi$, a gauge calibration can be performed as indicated. Then, an accurate determination of the electric field at each one of the filaments and therefore the local hysteretic losses may be truly achieved. 

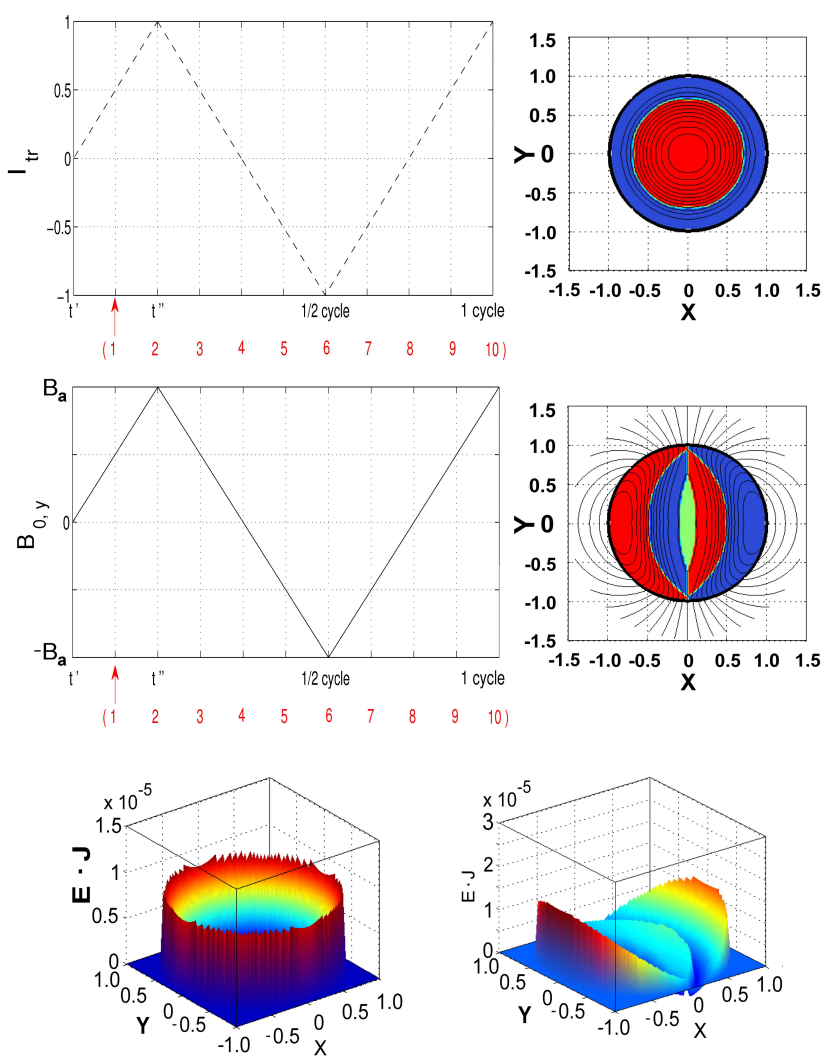

FIG. 1. (Color online) Sketch of some of the experimental processes analyzed along this paper. Here, a cylindrical SC wire of radius $\mathrm{R}$ is subjected to isolated triangular excitations $I_{t r}(t)$ (top pane) and $B_{y}(t)$ (middle pane) of amplitudes $I_{\mathrm{a}}$ and $B_{\mathrm{a}}$ respectively. The profiles of current density for the time-step labeled as 4 are displayed to the right, for the amplitudes $I_{\mathrm{a}}=1$ and $B_{\mathrm{a}}=6$ respectively. The bottom pane shows the corresponding profiles for the density of power dissipation in the aforementioned cases (left: transport current. Right: magnetic field). Hereinafter, units are $\left(\mu_{0} / 4 \pi\right) R J_{c}$ for $B_{0, y}$, and $I_{c} \equiv \pi R^{2} J_{c}$ for $I_{t r}$.

\section{RESULTS}

The electromagnetic behavior of a round superconducting wire subjected to the action of a transverse magnetic field $B_{y}(t)$ and a longitudinal transport current of intensity $I_{t r, z}(t)$ has been simulated in different conditions. The time dependence of the electromagnetic excitations $\left(B_{y}, I_{t r, z}\right)$ follows the triangular pattern shown in Fig. 1. This figure also shows the penetration profiles of current density and the magnetic field lines (isolevels of magnetic vector potential) for the corresponding isolated excitations, $I_{t r}$ (top pane) or $B_{y}$ (middle 


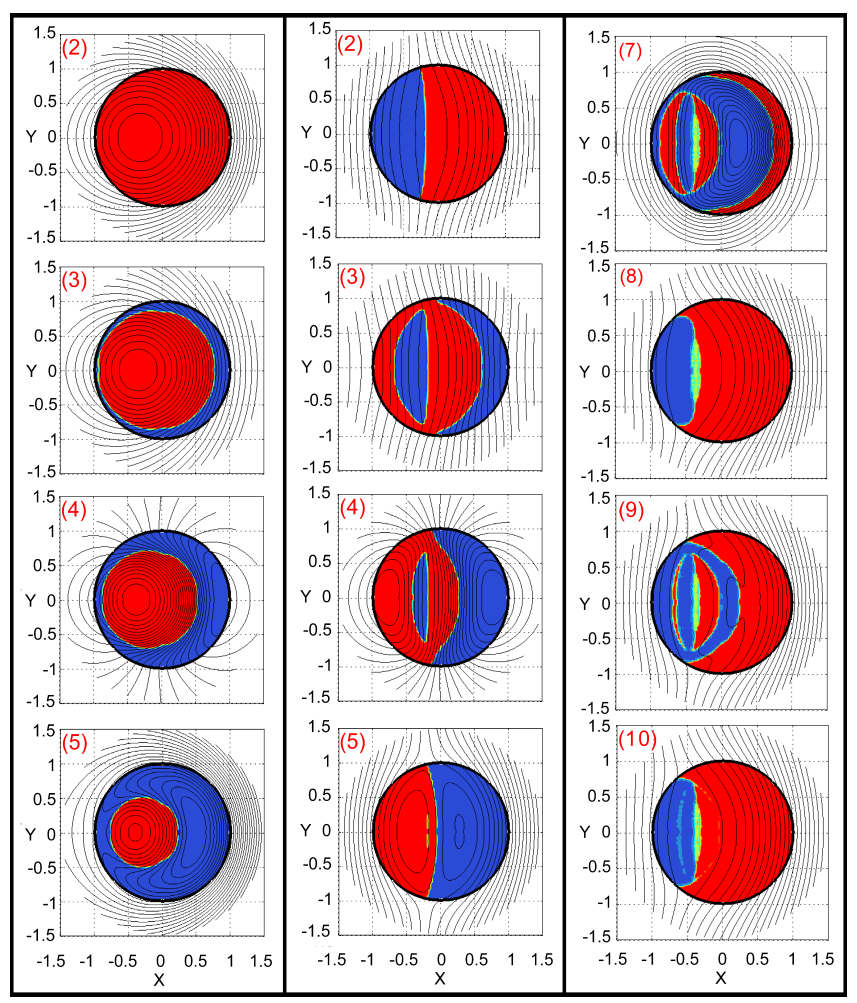

FIG. 2. (Color online) Magnetic flux lines and profiles of current density of a round superconducting wire subjected to the synchronous action of the electromagnetic sources depicted in Fig. 1, where the time-steps shown have been accordingly labeled. First two columns correspond to a virgin-wire subjected to synchronous sources of amplitudes $\left[I_{\mathrm{a}}=1, B_{\mathrm{a}}=2\right]$ (left pane), and $\left[I_{\mathrm{a}}=0.25, B_{\mathrm{a}}=8\right]$ (middle pane). In addition, a premagnetized wire with $B_{0, y}(t=0)=2$ has been considered previous to the action of the synchronous sources of amplitudes $\left[I_{\mathrm{a}}=0.5, B_{\mathrm{a}}=4\right]$.

pane). Trivially, for a long cylindrical wire only subjected to a transport current, the flux front is defined by a circumference of radius $\tilde{r}=R \sqrt{1-I_{t r} / I_{c}}$, which straightforwardly allows to obtain the analytical solution of the entire electromagnetic problem. However, for the seemingly simple case of the superconducting wire subjected to a transverse magnetic field, the flux-front cannot be defined by an exact analytical solution. Nevertheless, a number of approaches to characterize this boundary have been reported. ${ }^{9-12}$. Noteworthy is that the local density of power dissipation across the section of the superconducting wire follows the same symmetry defined by the flux front boundary, with its maximal intensity over the surface of the superconducting wire (see bottom pane of Fig. 1). Naively, in a first approximation, for describing the local electromagnetic properties of superconducting wires 
under configurations of simultaneous alternating current and applied magnetic field, one could argue that the above described patterns or their slight modifications: "current-like" or "field-like" tendencies may serve for extremal excitation amplitudes $I_{\mathrm{a}}$ and $B_{\mathrm{a}}$. Thus, within this simplified scenario, the flux-front would be depicted by a linear superposition of the profiles of current density for isolated excitations. For instance, one could start with the "field-like" shielding profiles and then apply a displacemen of its geometrical center as result of a kind of "Lorentz force" related to the application of a transport current. Fig.2 shows that this picture is basically correct.

On the other hand, in the light of this "superposition" ansatz, one would expect an axially symmetric distribution of density of power dissipation leading to a quasi-homogeneous heat release over the surface of the superconducting wire. This follows from the patterns displayed for the cases with isolated excitations (see Fig.1). However, as we have shown in Ref. ${ }^{8}$ neither the linear superposition of the isolated contributions can deal with an accurate evaluation of the hysteretic losses, nor with the local distribution of profiles of current density. Therefore, determining the distribution of heat release across the superconductor is far from being intuitive. As shown below, for diverse experimental configurations with a simultaneous action of an oscillating magnetic field and an ac transport current, the density of power dissipation across the section of the superconducting wire shows a strong localization towards one side of the wire during the entire cyclic process.

In order to prove the above statement, the power dissipation profiles corresponding to the processes in Fig. 2 are shown in Fig. 3. Notice the strong localization of the specific power density. This is revealed as a universal behavior for superconducting wires subjected to oscillating excitations, even at those cases where the superconducting wire has been magnetized previous to the cyclic oscillation stage (rightmost columns of Figs. 2 and 3).

Certainly, determining the flux front for synchronous electromagnetic excitations with low magnetic field is almost intuitive as the distribution of profiles of current density develops a current-like pattern (see left pane of Fig. 2). However, for high magnetic fields, ascertaining the distribution of profiles of current density in the cyclic stage is more elaborated, as long as the electromagnetic history is not erased by the maximal condition for the amplitude of the ac transport current $I_{\mathrm{a}}=I_{c}$. Actually, if $I_{\mathrm{a}}<I_{c}$ the flux fronts do not overlap to a unique contour line defined by the filaments with current alternating between $I_{c}$ and $-I_{c}$, although a field-like pattern may still be identified as it is observed from the current profiles depicted 


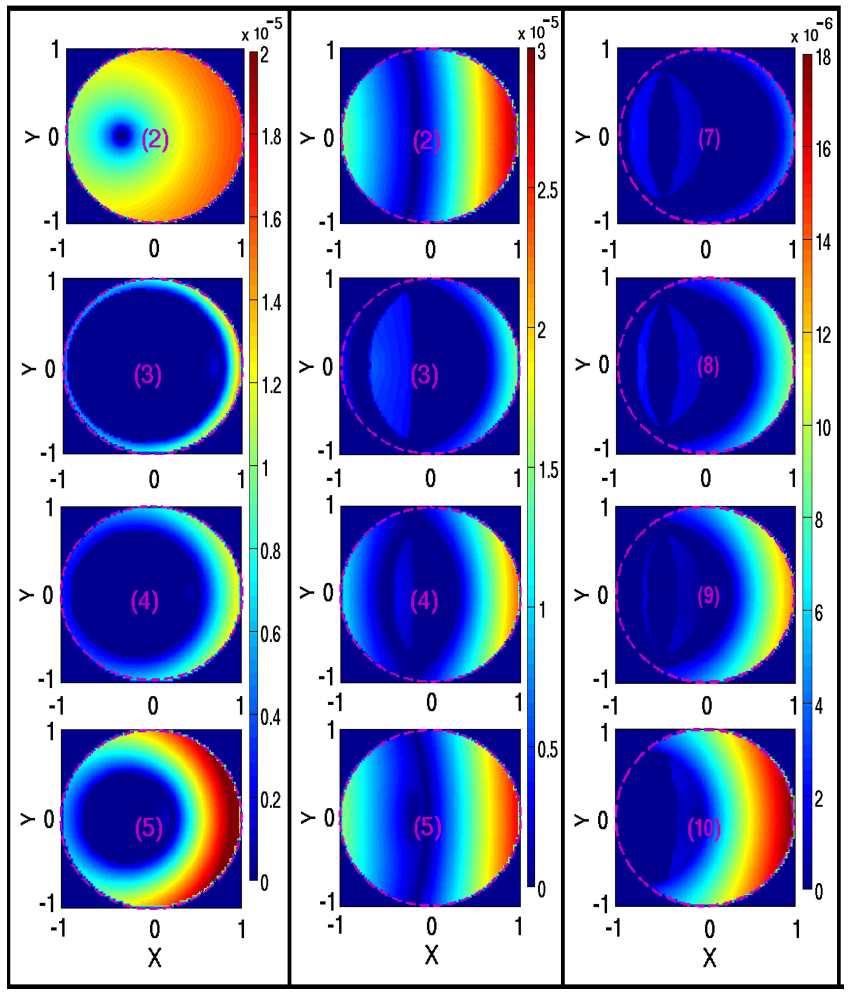

FIG. 3. (Color online) Local profiles for density of power dissipation $\mathbf{E} \cdot \mathbf{J}$ across the section of the superconducting wire, in correspondence to the profiles of current density displayed in Fig. 2. In the left pane, the colormap for the time-step labeled with a (2) must be read with a renormalization factor of 5 . Units are $\left(\mu_{0} / 4 \pi\right) R^{2} J_{c}^{2} / \delta t$ for $\mathbf{E} \cdot \mathbf{J}$.

in the second column of Fig. 2. Further, the definition of the flux front becomes even more tangled when premagnetized wires are considered, because multiple domains enclosed by the aforementioned contour lines arise (see right pane of Fig. 2).

Remarkably, despite the appearance of a wide variety of flux-fronts described above, we have observed that the local density of power dissipation $\mathbf{E} \cdot \mathbf{J}$ evolves in a rather defined pattern along the cyclic stage, with a strong localization of the heat release towards one of the sides of the wire, independently of the intensity of the synchronous oscillating excitations $B_{0}$ and $I_{t r}$. Notoriously, one can identify that the asymmetric distribution of power losses is straightforwardly linked to the zone where the the maximal density of magnetic flux occurs, which corresponds to the "active" zone where the filaments are carrying a current of intensity $I_{c}$ in the same direction of flow of the injected transport current $I_{t r}$. Thus, this asymmetric distribution of power losses remains along the entire cycle perpendicular to the orientation 
of $\mathbf{B}_{0}$ as long as both excitations evolve synchronously.

\section{CONCLUSION}

A systematic study of the local electromagnetic behavior of a round superconducting wire subjected to the simultaneous action of an oscillating magnetic field of amplitude $B_{\mathrm{a}}$ and a synchronous transport current of amplitude $I_{\mathrm{a}}$, has been pursued under the critical state model. Profiles of current density of the so-defined "current-like" type have been identified for high values of the transport current $\left(I_{\mathrm{a}} \simeq I_{c}\right)$, where the flux front shows a semi-circular shape shifted towards the direction of the unit vector $\hat{u}=\hat{\mathbf{B}} \times \hat{\mathbf{u}}_{t r}$, with $\hat{\mathbf{u}}_{t r}$ the direction of flow of the transport current. On the other hand, for low values of current $\left(I_{\mathrm{a}}<I_{c} / 4\right)$ and moderate or high values of field $\left(B_{\mathrm{a}}>4\right)$, profiles of current density of the "field-like" type may be identified by connecting the sharp corners of the multiple domains of current flow defined by filaments with currents alternating between $I_{c}$ and $-I_{c}$. Likewise, for premagnetized wires we have shown the occurrence of even more complex structures for the flux front, with the occurrence of multiple domains seemingly unconnected. The strong deformation of the classical flux fronts with circular or semi-elliptical symmetries does not allow a simple approach to the description of the local electromagnetic properties. However, we have identified a counterintuitive overall pattern in the heat release of the superconducting wire. A universal asymmetric distribution of the local power density losses towards one side of the cross section is found. This trend is independent of the intensity of the electromagnetic excitations $B_{0}$ and $I_{t r}$, as long as both excitations evolve synchronous. As a matter of fact, this strong localization of the density of power dissipation is a straightforward consequence of the underlying domain structure of the current flow, induced by the interaction of shielding and transport.

\section{ACKNOWLEDGMENT}

This work was supported by the Spanish MINECO and the FEDER program (MAT201122719 project), DGA grant T12/2011, and the Spanish CSIC JAE program. H. S. Ruiz 
thanks H. Rauh for helpful discussions.

1 C. P. Bean, "Magnetization of Hard Superconductors," Phys. Rev. Lett. vol. 8, pp. 250-253, March 1962.

2 C. P. Bean, "Magnetization of High-Field Superconductors," Rev. Mod. Phys., vol.36, pp. 31-39, 1964.

3 R. Hancox, "Calculation of a.c. losses in a type II superconductor" Proc. IEE, vol. 113, pp. $12211228,1966$.

4 A. Badía-Majós, C. López, and H. S. Ruiz, "General critical states in type-II superconductors," Phys. Rev. B, vol. 80, pp. 144509, 2009.

5 L. Prigozhin, "Variational inequalities in critical-state problems," Physica D, vol. 167, pp. 197$210,2004$.

6 E. Pardo, F. Gömöry, J. Šouc and J. M. Ceballos, "Current distribution and ac loss for a superconducting rectangular strip with in-phase alternating current and applied eld," Supercond. Sci. Technol., vol. 20, pp. 351-364, 2007.

7 H. S. Ruiz, and A. Badía-Majós, "Smooth double critical state theory for type-II superconductors," Supercond. Sci. Technol., vol. 23, pp. 105007, 2010.

8 H. S. Ruiz, A. Badía-Majós Y. A. Genenko, H. Rauh, and S.V. Yampolskii, "Superconducting wires under simultaneous oscillating sources: magnetic response, dissipation of energy and low pass filtering," Appl. Phys. Lett., vol. 100, pp. 112602, 2012.

9 W.J. Carr, Jr., M.S. Walker, and J.H. Murphy, "Alternating field loss in a multifilament superconducting wire for weak ac fields superposed on a constant bias," J. Appl. Phys., 46, pp. 4048, (1975).

10 V.B. Zenkevitch, A.S. Romanyuk, V.V. Zeltov, "Losses in composite superconductors at high levels of magnetic field excitation: part 1," Cryogenics 20, pp. 703 (1980).

11 W.J. Carr, AC loss and macroscopic theory of superconductors 2nd Ed. (Taylor \& Francis, NY, 2001).

12 A.V. Gurevich, R.G. Mints, A.L. Rakhmanov, Physics of composite superconductors, Nauka Publishers, 1987 in Russian) [Engl. translation: Begell House, NY, 1997]. 